\title{
Pectus Excavatum postsurgical outcome based on preoperative soft body dynamics simulation
}

\author{
António H.J. Moreira ${ }^{1,2}$, Pedro L. Rodrigues ${ }^{1,2}$, Jaime Fonseca $^{5}$, A.C.M. Pinho ${ }^{6}$, Nuno F. \\ Rodrigues $^{3,4}$, Jorge Correia-Pinto ${ }^{1,2}$, João L. Vilaça ${ }^{1,2,3}$ \\ ${ }^{1}$ Life and Health Sciences Research Institute, SHS, University of Minho, Portugal \\ ${ }^{2}$ ICVS/3B's - PT Government Associate Laboratory, Braga/Guimarães, Portugal \\ ${ }^{3}$ DIGARC - Polytechnic Institute of Cávado and Ave, Barcelos, Portugal \\ ${ }^{4}$ HASLab / INESC TEC - University of Minho, Braga, Portugal \\ ${ }^{5} \mathrm{DEI}$ - Industrial Electronics Department, University of Minho, Guimarães, Portugal \\ ${ }^{6}$ DEM - Mechanical Department, University of Minho, Guimarães, Portugal
}

\begin{abstract}
Pectus excavatum is the most common congenital deformity of the anterior chest wall, in which an abnormal formation of the rib cage gives the chest a caved-in or sunken appearance. Today, the surgical correction of this deformity is carried out in children and adults through Nuss technic, which consists in the placement of a prosthetic bar under the sternum and over the ribs. Although this technique has been shown to be safe and reliable, not all patients have achieved adequate cosmetic outcome. This often leads to psychological problems and social stress, before and after the surgical correction. This paper targets this particular problem by presenting a method to predict the patient surgical outcome based on pre-surgical imagiologic information and chest skin dynamic modulation. The proposed approach uses the patient pre-surgical thoracic CT scan and anatomical-surgical references to perform a 3D segmentation of the left ribs, right ribs, sternum and skin. The technique encompasses three steps: a) approximation of the cartilages, between the ribs and the sternum, trough b-spline interpolation; b) a volumetric mass spring model that connects two layers - inner skin layer based on the outer pleura contour and the outer surface skin; and c) displacement of the sternum according to the prosthetic bar position.

A dynamic model of the skin around the chest wall region was generated, capable of simulating the effect of the movement of the prosthetic bar along the sternum. The results were compared and validated with patient postsurgical skin surface acquired with Polhemus FastSCAN system.
\end{abstract}

Keywords: Pectus excavatum, 3D simulation, cosmetic outcome, soft body dynamics

\section{INTRODUCTION}

Pectus excavatum (PE) is characterized by an abnormal formation of the rib cage associated with diminished respiratory and altered cardiac functions resulting in the decreased ability of cardiovascular activities. This deformity presents an incidence rate of 1:1000 births, with a male predominance of 4 for 1 . Nowadays, two techniques are used for PE correction. One is the classic surgical technique developed in the 50's, called Ravitch technique and, more recently, the Nuss technique has been applied with considerable positive results.

Since the introduction of the Nuss technique in 1997, thousands of patients all over the world have been treated with this novel minimal invasive surgical correction [1-3]. The improved surgical correction of the chest deformity gives the patient a sense of self-image and worth, resulting in an enhanced quality of life [4]. Although this procedure is well known, it lacks sensibility in cases of sternum angulation or abnormal ribs growth, being difficult to predict the cosmetic outcome.

In 2009, Vilaça et al. developed a system for automatically modelling and bending the metal bar used in Nuss technique, allowing its pre-operative personalization according to the thoracic morphology of each patient. The size and

\footnotetext{
Medical Imaging 2012: Image-Guided Procedures, Robotic Interventions, and Modeling,

edited by David R. Holmes III, Kenneth H. Wong, Proc. of SPIE Vol. 8316, 83160K

(C) 2012 SPIE · CCC code: $1605-7422 / 12 / \$ 18 \cdot$ doi: $10.1117 / 12.911601$
}

Proc. of SPIE Vol. $831683160 \mathrm{~K}-1$ 
shape of the prosthesis is determined by the ribs profile, position and some anatomo-surgical references, acquired by computed tomography (CT) [5]. This system has already been tested successfully at Hospital S. Joao-Porto, Portugal.

Until now, automatic or manual bending of the metal bar is solely based on patient CT data or external mould respectively, without any feedback concerning cosmetic outcome aspects. In some cases, the prosthesis is manually modelled during the surgical procedure in order to improve the overall outcome. After the surgical procedure, the prosthesis is left in place from 24 up to 36 months (forcing the sternum to a new position), after which it is removed by reopening the thorax using small lateral incisions [6]. In this long process the best outcome is dependent on the curvature of the prosthesis for each patient, bar positioning and displacement $[7,8]$.

The correction procedure imposes two fundamental problems. First, the time span between the surgery and the removal of the prosthesis complicates the prediction of the patient tissues evolution around the prosthesis, especially in cases of young patients. Secondly, the patient itself is often reluctant in performing such a corrective procedure without any concrete results prediction - other than the one verbally or schematically given by the surgeon - of his final physical aspect at the end.

This problem could be solved by the introduction of virtual simulation tools to predict and improve the overall curvature of the prosthesis bar, improving the functional and visual outcome for each patient, and also enhancing their quality of life [9]. In particular, hard and soft body dynamics simulation could play an important role in this domain, allowing the surgeon to predict and improve the prosthesis curvature for best visual outcome.

Few studies have evaluated the mechanical influence in sternum and ribs after the prosthesis bar placement, and from these, none evaluated the cosmetic outcome [10]. Vilaça et al. evaluated the volume of influence after PE correction in 21 male patients using artificial neural networks. The results showed a mean volume of $152 \times 10^{3} \mathrm{~mm}^{3}$ affected after correction. However, no success was found in this work, because the training procedure of the artificial neural networks did not converge to a satisfactory mathematical model.

Wei et. al., developed a finite element model (FEM) of PE, based on a single patient (a boy) CT image data. The study showed that the bar placement could be improved by $1 \sim 6 \mathrm{~mm}$. But, the cosmetic outcome of the patient was not evaluated. FEM's are widely used in biomechanics, where they have been capable of evaluating complex geometries with precision. Nevertheless, they are computationally slow, requiring complex model parameterization and the model extraction is highly time consuming.

This work proposes a novel method to simulate the patient thoracic skin resorting only to chest dynamic modulation through a mass-spring system [11, 12]. It allows the determination of the correct bar position/shape to minimize the caved-in or sunken appearance in the chess wall. The development of this tool has two distinct objectives: i) the development of a graphical software application to help the surgeon evaluate the expected cosmetic result of placing the thoracic prosthesis in different substernal positions; and, ii) help the patient decide or not for the surgical intervention, by evaluating a comprehensive and personalized simulation of the surgery outcome. The second objective is crucial, since many cases of surgical correction are only performed towards improvement of physical appearance.

The paper is divided as follows: section II describes the developed algorithms to extract the skin, ribs and sternum 3D information along the thoracic wall, the mass-spring model and the constraints connections between layer and ribs; and the definition of the sternum rotation axis and skin surface acquisition after PE correction. Section III presents the results of tissue variations compared with the original skin from CT and the scanned skin. Finally section IV presents the study conclusion and future developments.

\section{METHODS}

To achieve a dynamic modulation of the chest wall that allows prediction of the correct bar position that minimizes the caved-in appearance and maximizes the physical appearance, it was necessary to reconstruct several internal structures from CT images, such as sternum, ribs, inner and outer skin layer. This was achieved through image processing methods, implemented with $\mathrm{C}++/ \mathrm{OpenGL}$, which allowed the detection of ribs central points, sternum outline and fat tissue area. Subsequently, the mass-spring system is parameterized using the extracted two layer skin model and connected to the internal structures (ribs and sternum). This system will simulate the surface skin outcome.

The original skin surface and the 3D surface scan after the patient surgery are then compared using a mesh measurement algorithm with the simulation output.

In the end, a user interface was also developed, enabling fast DICOM images loading and allowing the placement of the prosthesis bar in different locations, to evaluate the displacement of sternum and skin remodelling. 


\subsection{Structures extraction}

Through image processing algorithms several slices from the patient CT scan containing the first thoracic rib up to the twelfth rib of the thoracic wall are analysed to segment the ribs, sternum contours and further allow the reestablishment of the cartilages between.

The segmentation process starts with an iso-contour algorithm based in Hounsfield value for bone contour determination, (see Figure 1a). Then, the ribs contours centroids coordinates are extracted using $\mathrm{Hu}$ invariant moments [13], in each CT slice (red points in Figure 1b). The cartilages are established as b-splines using the ribs contours centroids and the sternum cartilages connection points (white points in Figure 1c). B-Splines were chosen due to their smoothing properties, finer shape control and good overall curve result when comparing with real cartilages. The bspline sequence of control points is determined from the first slice, defining seed points on each centroid and afterwards calculating the root mean square value from each seed to the next slice. This step makes possible the definition of smooth control points for use in the mass-spring system affecting the skin surface.

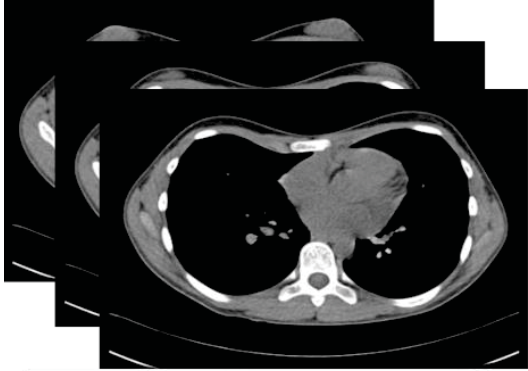

a) CT Scan

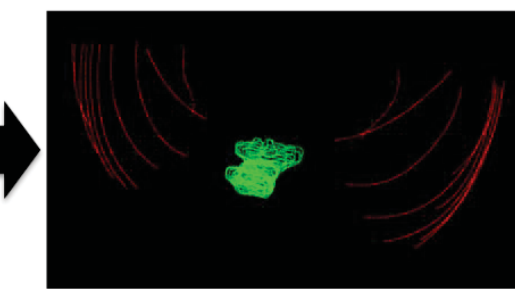

b) Ribs and Sternum

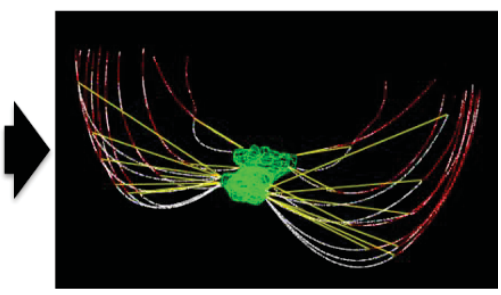

c) B-Spline Cartilage

Figure 1 - Reestablishment of the cartilages using B-Splines: a) CT scan data; b) ribs centroid (red points) and sternum (green points); and, c) B-spline reconstructed cartilages

\subsection{Mass-Spring Model}

The patient skin is modulated as a two layer volumetric mass-spring system, the inner layer connected to the internal structures and the outer layer (skin surface). For that, in each axial image slice, determined in the previous step, a Gaussian filter (standard deviation $=2.5$ ) was applied. Subsequently, all CT images showed up with a black background colour, improving the image contours detection using a Sobel operator. Then, all image pixels were vertically and horizontally tracked by recursively selecting all first pixels belonging to image gradient magnitude and taking into account only the points above the coronal plane. The result of this procedure was the skin substructure showed in Figure 2 (grey points). Secondly, the same process was applied to detect the inner skin layer (pleura), tracking the inverse gradient transition. With these two structures a relation between points is required to achieve the necessary interaction.

For that, mass-spring surfaces are modelled as a set of point masses connected by ideal weightless elastic and linear springs following Hook's law (Eq. 1), these springs give a linear relationship between forces applied on the skin points and the difference between the resting distance and the actual distance of the skin points.

$$
F=-k \cdot x
$$

Furthermore associated with the Newton's second law of movement, this equation describes the forces applied in one $3 \mathrm{D}$ point (Eq. 2):

$$
m_{i} \ddot{x}_{\imath}=-y_{i} \dot{x}_{\imath}+\sum_{j} g_{i j}+f_{i}
$$

The differential equation is then solved with a standard numerical method, the Verlet integration [14] (Eq. 3).

$$
x(t+h)=2 x(t)-x(t-h)+\ddot{x}(t) h^{2}
$$

For each point in the outer skin layer, several spring constraint connections were performed to achieve stability and smoothness between the two layers forming the volumetric skin, such as direct, stretch, shear, and bend springs (see 
Figure 2a). Each point of the outer skin is connected to a total of 49 neighbourhood points (25 to the inner layer and other 24 to the outer layer), Figure $2 b$.

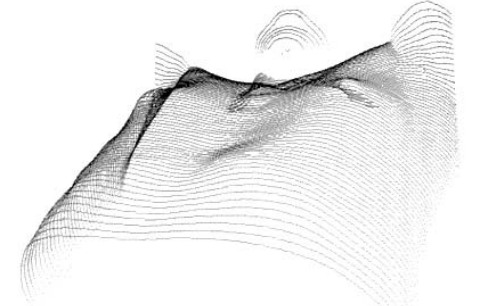

a) Skin points

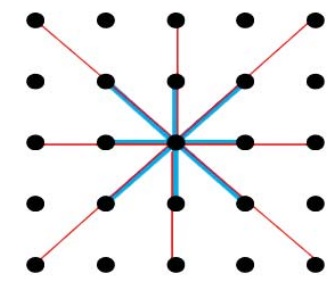

b) Skin points constrains

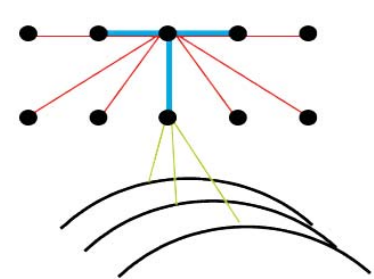

c) Inner layer connections

Figure 2 - Mass-spring system constrains: a) Skin layer points extracted from CT scan; b) Outer and inner layer skin constrains; c) Volumetric constrains connecting the skin layers and ribs.

In the inner skin mesh, each point is connected to the closest three independent ribs, cartilages and the sternum. In this way adjustments take place at the cartilage spline control points, e.g. moving the sternum to different positions Figure 2c, will generate changes in the inner skin surface points through the mass-spring model and then propagate smoothly to the outer skin layer.

\subsection{Sternum relocation}

The sternum contour is defined as a point cloud, extracted from the CT data and with a fixed axis rotation point on the manubrium bone, allowing the correct realignment of sternum with the ribs, Figure $3 \mathrm{a}$. In each rotation of the sternum, the b-spline representing ribs and sternum are recalculated and the springs connected are also updated.

The initial location for evaluation is defined as in Vilaça et al. system [5], determining that the prosthesis bar is placed on the lowest depression point of the sternum and between the intercostal space. If results are not satisfactory, further locations can be evaluated. The result of the modification of the surface points simulating PE correction are showed in Figure 3b.

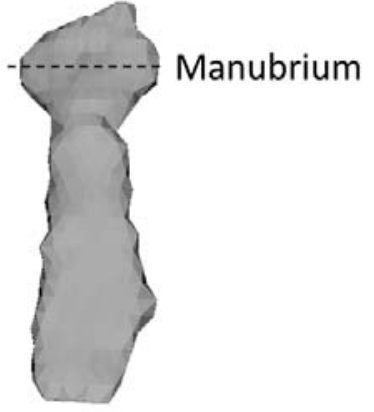

a) Rotation axis

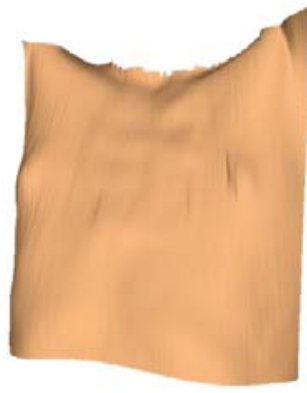

b) Simulated skin

Figure 3 - Sternum rotation and simulated skin surface; a) Axis of rotation centred on manubrium; b) Simulated chess wall skin surface after relocation of sternum to the expected position after surgery.

\subsection{Chest wall scan}

A portable 3D scanner (Polhemus FastScan) capable of creating instant real time 3D images was used to acquire the patient's anterior chest wall as an unstructured cloud-point. This system integrates a track unit to determine the position and orientation of a laser line on the object while a camera detects the laser to record cross-sectional depth profiles. In order to reduce errors caused by the patient respiratory movements in the data acquisition, the system reference was attached to the $10^{\circ}$ left rib.

After acquisition the surface reconstruction was generated through Radial Basis Functions (RBF) as in [9]. The patients observed in this validation process had placed the prosthesis bar between three to six months before the 3D surface scan.

Given the two meshes (simulated and 3D scan) in arbitrary initial poses, a set of four pairs of points were chosen as follows (Figure 4b): one on the neck, one on navel (if available) and one on each breast nipple. Using these points a 3D affine surface registration was performed to match the patient surfaces as close as possible. 


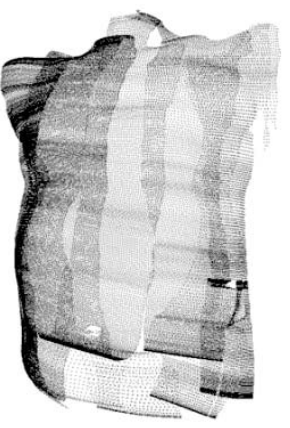

a) Cloud point

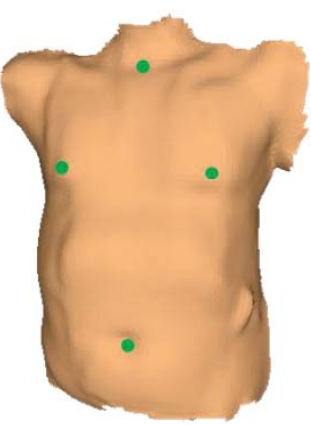

b) Surface reconstruction and registration points

Figure 4 - Chess wall surface scan and registration points; a) Reconstruction of the chess wall from an unstructured cloud-point; b)

Definition of 4 points for surface registration between the 3D scan, the simulated and TAC skin.

Finally, for all patients the simulated mesh was compared to the original and the scanned mesh, using the software CouldCompare [15].

In order to assist the surgeon in the decision process in the previously described simulation, a user interface was also developed, which encapsulates all the algorithms and allows the surgeon to (1) load the patient DICOM data, (2) automatically extract the bone structure, sternum contour and generate the b-spline approximation and also (3) enable the fast simulation of the skin surface when the sternum is rotated.

\section{RESULTS}

This soft body dynamics simulation methodology was evaluated in 3 patients with different severity of pectus excavatum acquired as described in section 2.1 and 2.4 at the Life and Health Sciences Research Institute (ICVS University of Minho) and Hospital S. Joao-Porto, Portugal. For all patients the surface skin form the CT scan was extracted, followed by the 3D surface scan after surgery. Both surfaces are compared to the simulated surface skin initially established from the CT scan. The 3D surface scan is previously registered to the simulated skin as described in section 2.4. Table I summarizes the averages errors between surfaces of all 3 male patients.
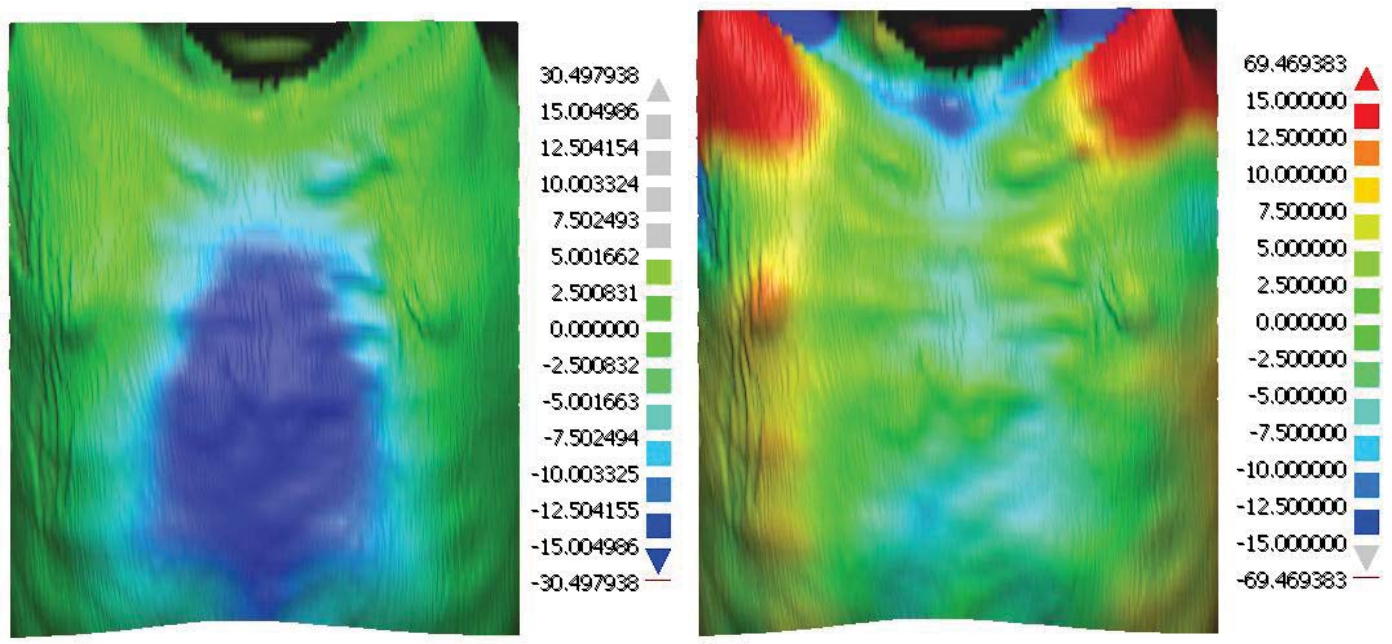

Figure 5 - Patient 1 comparative distance map between a) TAC scan and simulated skin surface; b) 3D surface scan and simulated skin surface. All values in millimetres. 

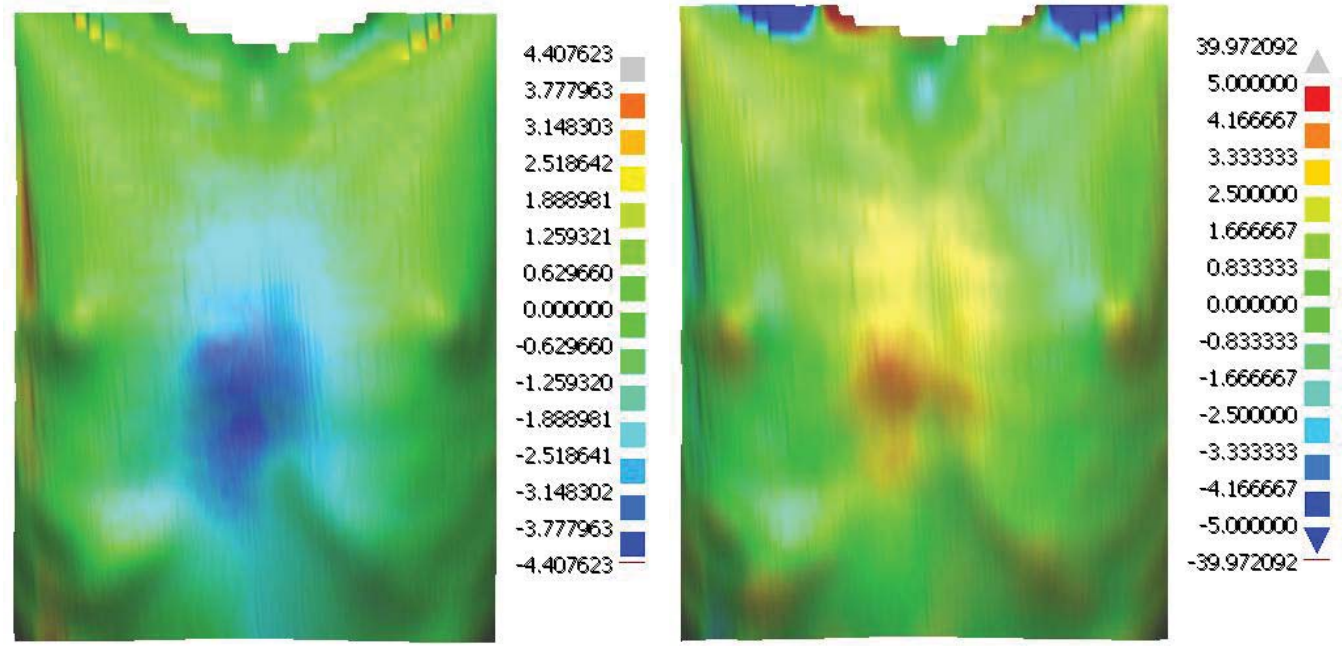

Figure 6 - Patient 2 comparative distance map between a) TAC scan and simulated skin surface; b) 3D surface scan and simulated skin surface. All values in millimetres.
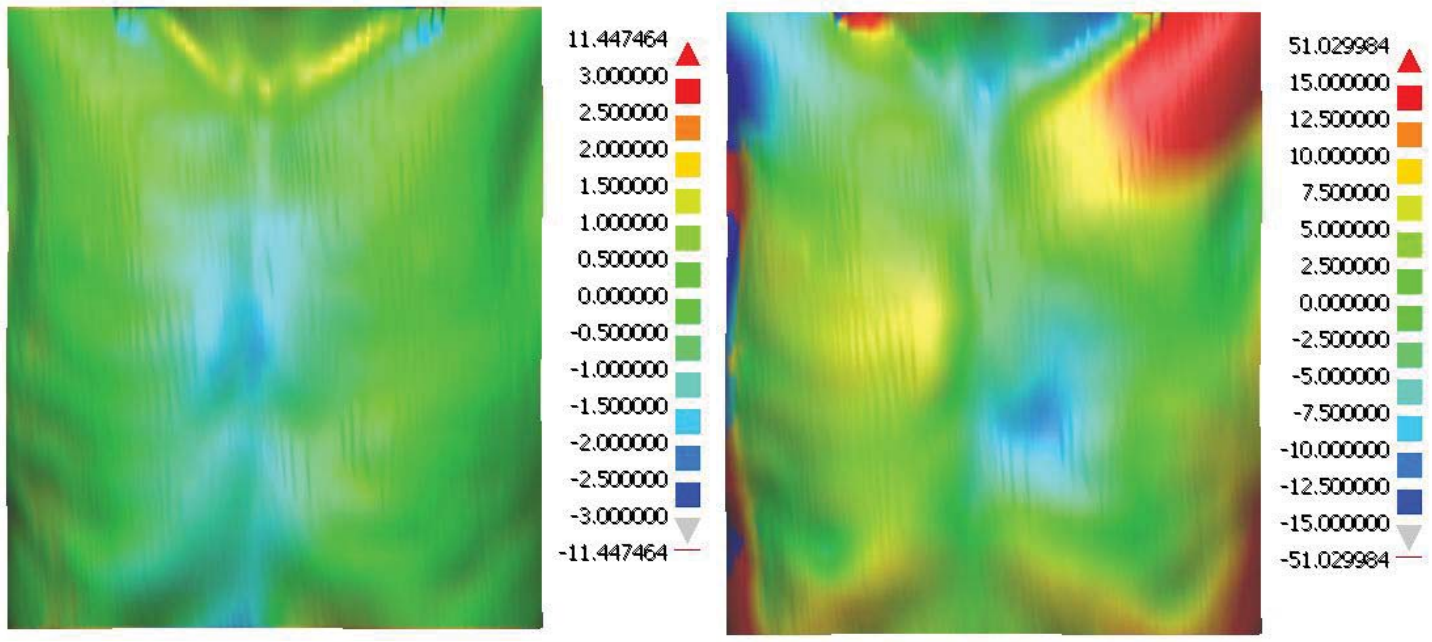

Figure 7 - Patient 3 comparative distance map between a) TAC scan and simulated skin surface; b) 3D surface scan and simulated skin surface. All values in millimetres.

Table 1 - Results summary - Differences between meshes

\begin{tabular}{|c|c|c|c|c|c|c|c|c|}
\cline { 2 - 9 } \multicolumn{1}{c|}{} & \multicolumn{3}{c|}{ CT mesh (before surgery) vs Simulated } & \multicolumn{3}{c|}{ 3D surface scan (after surgery) vs } \\
\cline { 2 - 9 } & $\begin{array}{c}\text { Mean } \\
\text { Distance } \\
(\mathrm{mm})\end{array}$ & $\begin{array}{c}\text { Standard } \\
\text { Deviation } \\
(\mathrm{mm})\end{array}$ & $\begin{array}{c}\text { Min } \\
(\mathrm{mm})\end{array}$ & $\begin{array}{c}\text { Max } \\
(\mathrm{mm})\end{array}$ & $\begin{array}{c}\text { Mean } \\
\text { Distance } \\
(\mathrm{mm})\end{array}$ & $\begin{array}{c}\text { Standard } \\
\text { Deviation } \\
(\mathrm{mm})\end{array}$ & $\begin{array}{c}\text { Min } \\
(\mathrm{mm})\end{array}$ & $\begin{array}{c}\text { Max } \\
(\mathrm{mm})\end{array}$ \\
\hline Patient 1 & -4.916 & 6.964 & -30.497 & 4.932 & 3.391 & 11.331 & -37.733 & 69.469 \\
\hline Patient 2 & -0.581 & 1.141 & -3.534 & 4.407 & 0.4163 & 2.610 & -39.970 & 8.196 \\
\hline Patient 3 & -0.164 & 0.701 & -8.304 & 11.447 & 3.341 & 9.609 & -41.587 & 51.029 \\
\hline
\end{tabular}

One of the main limitations of this work is the parameterization of the mass-spring system in the outer skin layer to achieve smoother transitions. This limitation was minimized with the introduction of an inner skin layer connected with a 
mass-spring system, creating a volumetric mass-spring model. The result of the b-spline rib and cartilage approximation was then input to this model and further constrains added to each inner skin point connecting with the $b$-spline and sternum points.

Another issue of this work is the lack of reference points and the dissimilar position between CT scan and 3D surface scan. As shown in Figure 5 and 7 the red areas exhibit high displacement between the simulated skin and the 3D surface skin acquired with the portable scanner, resulting in high min and max values in table I. Patients growing can also affect the overall results, in this particular case, patient 2 (see Figure 6) 3D surface scan was acquired only 2 months after surgery, demonstrating good results in the surface registration.

Within the limitations of this project, preliminary results show a mean error ranging from 0.41 to $3.39 \mathrm{~mm}$ when compared with the 3D surface scan.

\section{CONCLUSIONS}

This paper presents a study - involving 3 male patients - of a simulation model based in a mass-spring system of the thoracic wall surface after pectus excavatum surgical correction, comparing the distance errors between simulated skin, the CT skin and the acquired 3D surface skin.

Since most of pectus excavatum surgery is performed as cosmetic purpose, the introduction of virtual simulation tools to predict and improve the overall curvature of the prosthesis bar, will improve the functional and visual outcome, and help today's practice to further enhance the quality of life of each patient.

As so, this novel method to predict the patient surgical outcome based on the patient pre-surgical imagiologic information and skin dynamic modulation will help increase the acceptance of pectus excavatum surgery intervention and decrease the post-surgical corrections. Although this methodology works well for this set of patients, a vast set of new patients is needed to prove the effectiveness of this approach, as also improve the mass-spring system design.

Although more tests are need, this application can provide valuable feedback on the possible improvements in the prosthesis bar modulation to improve the postsurgical outcome. Future research paths also comprise the development and analysis of different fast soft body dynamical models such as energy minimization or finite element methods as a more physically accurate approach.

\section{ACKNOWLEDGEMENTS}

The authors acknowledge to Foundation for Science and Technology (FCT) - Portugal for the fellowships with the references: SFRH/BD/74276/2010; SFRH/BD/68270/2010; and, SFRH/BPD/46851/2008. This work was also supported by FCT R\&D project PTDC/SAU-BEB/103368/2008.

\section{REFERENCES}

[1] Hebra, A., "Minimally invasive repair of pectus excavatum", Semin Thorac Cardiovasc Surg, 21(1), 76-84 (2009).

[2] Iida, H., "Surgical repair of pectus excavatum", Gen Thorac Cardiovasc Surg, 58(2), 55-61 (2010).

[3] Nuss, D., et al., "A 10-year review of a minimally invasive technique for the correction of pectus excavatum", J Pediatr Surg, 33(4), 545-552 (1998).

[4] R.E. Kelly, T.F. Cash and R.C. Shamberger, et al. "Surgical repair of pectus excavatum markedly improves body image and perceived ability for physical activity: multicenter study" Pediatrics, 122(6), 1218-22 (2008)

[5] Vilaça, J L., Pinho, A., Correia-Pinto, J., Fonseca, J., Peixinho, N., "System for automatic and personalized modelling/bending of surgical prosthesis for correction of pectus excavatum based on pre-surgical imagiology information", WO2009/035358, (2009).

[6] Mao, Y.-Z., Tang, S.-T., Wang, Y., Tong, Q.-S., Ruan, Q.-L. "Nuss operation for pectus excavatum: a singleinstitution experience" World Journal of Pediatrics 5, 292-295 (2009).

[7] Hebra A., Swoveland B., Egbert M., Edward P. Tagge, Keith Georgeson, H. Biemann Othersen Jr., Donald Nuss, "Outcome analysis of minimally invasive repair of pectus excavatum: Review of 251 cases", Journal of Pediatric Surgery, 35(2), 252-258 (2000).

[8] Nagaso, Tomohisa, Miyamoto, Junpei, Kokaji, Kiyokazu, Yozu, Ryohei, Jiang, Hua, Jin, HongMei, Tamaki, Tamotsu "Double-bar application decreases postoperative pain after the Nuss procedure" J Thorac Cardiovasc Surg, 140, 39-44 (2010). 
[9] João L. Vilaça, António H. J. Moreira, Pedro L-Rodrigues, Nuno Rodrigues, Jaime C. Fonseca, A. C. M. Pinho and Jorge Correia-Pinto, "Virtual simulation of the postsurgical cosmetic outcome in patients with Pectus Excavatum", Proc. SPIE 7964, 79642L (2011)

[10] Wei Yanbin; Sun Deqiang; Liu Peng; Gao Ya; , "Pectus Excavatum Nuss Orthopedic finite element simulation," 3rd International Conference on Biomedical Engineering and Informatics (BMEI), 3, 1236-1239 (2010)

[11] Nebel and J.-C., 2000 Nebel, J.-C., "Soft Tissue Modelling From 3D Scanned Data", In Proceedings of Deform., 85-97 (2000).

[12] Wouter Mollemans, Filip Schutyser, Johan Van Cleynenbreugel, and Paul Suetens, "Tetrahedral Mass Spring Model for Fast Soft Tissue Deformation” N. Ayache and H. Delingette (Eds.), LNCS 2673, 145-154, (2003).

[13] Ming-Kuei Hu, "Pattern recognition by moment invariants". Proceedings of the IRE, 49, 1428 (1961).

[14] Mosegaard J., Herborg P., and Thomas S. Sørensen. "A GPU accelerated spring mass system for surgical simulation." Studies in health technology and informatics, 111, 342-348 (2005).

[15] CloudCompare (version 2.3), [GPL software]. EDF R\&D, Telecom ParisTech (2011). Retrieved from http://www.danielgm.net/cc/ 